\title{
Effective Detuning of a Three-Level Atom Interacted with the Laguerre-Gaussian Laser Beam
}

\author{
Yanli Wang ${ }^{1}$, Zhengling Wang ${ }^{1, \mathrm{a}}$, Naifei Ren ${ }^{2}$, Yaru Wang ${ }^{1}$, Xinzhi Hu${ }^{1}$ \\ ${ }^{1}$ Faculty of Science, Jiangsu University, Zhenjiang 212013, China \\ ${ }^{2}$ School of Mechanical Engineering, Jiangsu University, Zhenjiang 212013, China \\ azlwang@ujs.edu.cn
}

\begin{abstract}
We derive the general analytic expression of the effective detuning of the linearly polarized light field from the $\Lambda$-configuration three-level atomic mode. The detuning amending term resulting from the three-level atomic mode is not related with the type of light field, which is determined by the two lower level split and the ratio of the two lower level dipole matrix element to the total dipole matrix element in the three-level atomicmode. For a linearly polarized Laguerre-Gaussian laser beam, we show that there is same frequency amending for the axial, radial and azimuthal frequency detuning. We define an effective two-level atomic mode to substitute the three-level atomic mode, in which it can be more practical and convenient in consideration of the light detuning and Doppler shift from atomic transition frequency.
\end{abstract}

Keywords-three-level atomic mode, laguerre-Gaussian laser beam, light detuning, Doppler shift

\section{INTRODUCTION}

In 1936, Beth first experimentally observed the deflection of a quartz wave plate suspended from a thin quartz fiber with circularly polarized light passing through it [1]. In 1992, Allen et al. observed the torque on suspended cylindrical lenses arising from the reversal of the helicity of a Laguerre-Gaussian mode with welldefined orbital angular momentum [2]. Recently, the transfer of angular momentum from a light beam carrying angular momentum to absorbing macroscopic particles trapped in an optical tweezer causing them to rotate has been experimentally and theoretically studied [3-6]. Furthermore, an atom moving in a Laguerre-Gaussian beam experiences a torque and an azimuthal shift in its resonant frequency in addition to the usual axial Doppler shift and recoil shift [7-8]. Where they considered a twolevel atom which is interacted with the Laguerre-Gaussian beam and obtained the axial, radial and azimuthal Doppler shift as a complementarity for the total detuning of the beam frequency from the atomic resonant frequency.

In this paper, we extend the two-level atomic mode to the three-level atomic mode because the three-level atom has been received considerable attention for creating many new quantum effects [9-10]. First we consider a $\Lambda$ configuration three-level atom in a light beam and derive the general expression of the detuning of the beam from the three-level atom. Then taking a linearly polarized
Laguerre-Gaussian for an example, we derive the total detuning of the Laguerre-Gaussian beam from the threelevel atom. Finally, for the three-level atomic mode we derive an effective detuning and an effective two-level atomic mode.

\section{CALCUlation AND Discussion}

We consider a $\Lambda$-configuration three-level atom with one upper level $|3\rangle$ and two lower levels $|1\rangle,|2\rangle$ with frequency split $\Delta_{\mathrm{g}}$, as shown in Fig.1, which is interacted with an arbitrary light field with the frequency $\omega_{L}$. The atomic transition between $|1\rangle$ and $|3\rangle,|2\rangle$ and $|3\rangle$ are assumed to be optically allowed, while the transition between $|1\rangle$ and $|2\rangle$ is forbidden, which can be resulted from the $\mathrm{D}_{1}, \mathrm{D}_{2}$ line of alkali-metal atoms.

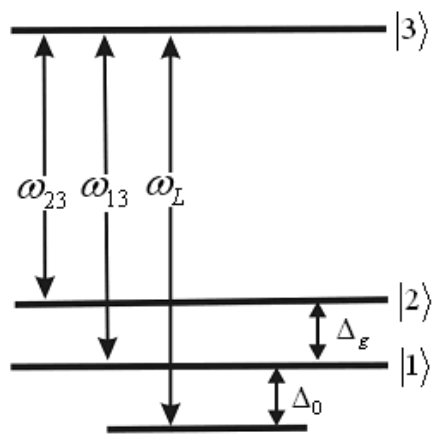

Figure 1 . The $\Lambda$-configuration three-level atom diagram

The Hamiltonian of our system is written as [9] $H=\mathbf{P}^{2} / 2 m+U(\mathbf{R})+\hbar \omega^{\prime}|1\rangle\left\langle 1\left|+\hbar \omega_{2}^{\prime}\right| 2\right\rangle\left\langle 2\left|+\hbar \omega_{3}^{\prime}\right| 3\right\rangle\langle 3|+\hbar \omega_{L} a^{+} a+H_{\text {int }}$

Where $\mathbf{P}$ and $\mathbf{R}$ are the momentum vector and the position vector of the atomic center of mass with atomic mass $m$, and $U(\mathbf{R})$ is trapping potential. Where $\hbar \omega_{1}^{\prime}, \hbar \omega_{2}^{\prime}$, $\hbar \omega_{3}^{\prime}$ are eigenenergys of levels $|1\rangle,|2\rangle,|3\rangle$, respectively, and $\omega_{13}=\omega_{3}^{\prime}-\omega_{1}^{\prime}, \omega_{23}=\omega_{3}^{\prime}-\omega_{2}^{\prime}$ are atomic transition frequencies. $a^{+}\left(a^{-}\right)$is the creation (annihilation) operator of light field $\omega_{L}$. The detuning of the laser frequency 
from atomic transition $\omega_{13}$ is $\Delta_{0}$. The last part $H_{\text {int }}$ is the interaction Hamiltonian, taking electronic dipole and rotating wave approximating, which in the interaction picture may be given by

$$
\begin{aligned}
H_{\mathrm{int}}= & -i \hbar\left\{e^{-i \Delta_{0} t} \pi^{+} a f_{1}(\mathbf{R}, t)-f_{1}^{*}(\mathbf{R}, t) a^{+} \pi e^{i \Delta_{0} t}\right\} \\
& -i \hbar\left\{e^{-i\left(\Delta_{0}+\Delta_{g}\right) t} \sigma^{+} a f_{2}(\mathbf{R}, t)-f_{2}^{*}(\mathbf{R}, t) a^{+} \sigma e^{i\left(\Delta_{0}+\Delta_{g}\right) t}\right\}
\end{aligned}
$$

Where transition operators $\hat{\pi}^{+}$and $\hat{\sigma}^{+}$represent transitions from $|1\rangle$ to $|3\rangle$ and $|2\rangle$ to $|3\rangle$ respectively, while transition operators $\hat{\pi}$ and $\hat{\sigma}$ represent transitions from $|3\rangle$ to $|1\rangle$ and $|3\rangle$ to $|2\rangle$ respectively. The coupling parameters $f_{1}(\mathbf{R}, t)$ and $f_{2}(\mathbf{R}, t)$ can be written as

$$
\begin{gathered}
f_{1}(\mathbf{R}, t)=G_{1}(\mathbf{R}) e^{i\left[\theta(\mathbf{R})+\Delta_{0} t\right]}, \\
f_{2}(\mathbf{R}, t)=G_{2}(\mathbf{R}) e^{i\left[\theta(\mathbf{R})+\left(\Delta_{0}+\Delta_{g}\right) t\right]} .
\end{gathered}
$$

Where $G_{1}(\mathbf{R})=\frac{1}{\hbar} \mathbf{D}_{13} \cdot \boldsymbol{\varepsilon}(\mathbf{R})$ and $G_{2}(\mathbf{R})=\frac{1}{\hbar} \mathbf{D}_{23} \cdot \boldsymbol{\varepsilon}(\mathbf{R})$ correspond to position dependent Rabi frequency, $\boldsymbol{\varepsilon}$ and $\theta(\mathbf{R})$ are the light field amplitude and phase respectively, $\mathbf{D}_{13}\left(\mathbf{D}_{23}\right)$ is the dipole matrix element between levels $|1\rangle$ $(|2\rangle)$ and $|3\rangle$. The total coupling parameter $f(\mathbf{R}, t)$ can be written as

$$
f(\mathbf{R}, t)=f_{1}(\mathbf{R}, t)+f_{2}(\mathbf{R}, t) .
$$

The time evolution of the total coupling parameter is derivable from the Heisenberg equation of motion, which is given as [7]

$$
\frac{d f(\mathbf{R}, t)}{d t}=\frac{i}{\hbar}[H, f(\mathbf{R}, t)]=\frac{1}{2 m}(\mathbf{P} \cdot \nabla f(\mathbf{R}, t)+\nabla f(\mathbf{R}, t) \cdot \mathbf{P}) .
$$

Here we have assumed that the coupling parameter $f_{1}(\mathbf{R}, t)$ and $f_{2}(\mathbf{R}, t)$ commute with $U(\mathbf{R})$.

From Eq.(5), we may derive the frequency detuning operator $\delta$ including Doppler and recoil frequency shift, etc, which by considering the leading order is given by[7]

$$
\delta=-\frac{i}{2 m}\left(\frac{\mathbf{P} \cdot \nabla f(\mathbf{R}, t)+\nabla f(\mathbf{R}, t) \cdot \mathbf{P}}{f(\mathbf{R}, t)}\right)_{0} .
$$

Here the subscript zero denotes operators at the initial time $t=0$. Eq.(6) can be further written as

$$
\delta=\left[-i \frac{\nabla f(\mathbf{R}, t) \cdot \mathbf{v}}{f(\mathbf{R}, t)}-\frac{\hbar \nabla^{2} f(\mathbf{R}, t)}{2 m f(\mathbf{R}, t)}\right]_{0} .
$$

For the case of a linearly polarized along the $x$-axis plane wave, we assume $\mathbf{D}_{13}=D_{13} \hat{\mathbf{x}}, \quad \mathbf{D}_{23}=D_{23} \hat{\mathbf{x}}$, $\boldsymbol{\varepsilon}(\mathbf{R})=\varepsilon \hat{\mathbf{x}}, \theta(\mathbf{R})=-\mathbf{k} \cdot \mathbf{R}$, and obtain

$$
\left[-i \frac{\nabla f(\mathbf{R}, t) \cdot \mathbf{v}}{f(\mathbf{R}, t)}\right]_{0}=\Delta_{0}-\mathbf{k} \cdot \mathbf{v}+\frac{D_{23}}{D_{13}+D_{23}} \Delta_{g}
$$

$$
\begin{aligned}
{\left[-\frac{\hbar \nabla^{2} f(\mathbf{R}, t)}{2 m f(\mathbf{R}, t)}\right]_{0}=} & \frac{\hbar k^{2}}{2 m}-\left(\frac{\hbar k \Delta_{0}}{m v}+\frac{\hbar k}{m v} \frac{D_{23} \Delta_{g}}{D_{13}+D_{23}}\right)+\frac{\hbar \Delta_{0}{ }^{2}}{2 m v^{2}} \\
& +\frac{\hbar\left(2 \Delta_{0} \Delta_{g}+\Delta_{g}{ }^{2}\right)}{2 m v^{2}} \frac{D_{23}}{D_{13}+D_{23}}
\end{aligned}
$$

We know from Eqs.(8.a), (8.b) that there are amending terms resulting from three-level atomic mode and related with two lower level split $\Delta_{g}, \mathrm{D}_{13}$ and $\mathrm{D}_{23}$ for the

Doppler shift $-\mathbf{k} \cdot \mathbf{v}$ and recoil shift $\hbar k^{2} / 2 m$.

Then we consider the case of atoms moving in a Laguerre-Gaussian beam which can be readily produced in the laboratory [10]. In the paraxial approximation, assuming the Laguerre-Gaussian beam traveling in the $-z$ direction and polarized in the $x$ direction and $\mathbf{D}_{13}=D_{13} \hat{\mathbf{x}}$, $\mathbf{D}_{23}=D_{23} \hat{\mathbf{x}}$, we derive the total coupling parameter $f(\mathbf{R}, t)=f_{1}(\mathbf{R}, t)+f_{2}(\mathbf{R}, t)$, and $f_{1}(\mathbf{R}, t), f_{2}(\mathbf{R}, t)$ are expressed as

$f_{1}(\mathbf{R}, t)=-i \omega_{L} D_{13} u_{p l}(r, z) \exp (-i k z) \exp (-i l \phi) \exp \left(i \Delta_{0} t\right)$

$f_{2}(\mathbf{R}, t)=-i \omega_{L} D_{23} u_{p l}(r, z) \exp (-i k z) \exp (-i l \phi) \exp i\left(\begin{array}{c}(9 . \mathrm{a}) \\ \left(\Delta_{0}+\Delta_{g}\right) t\end{array}\right.$

where the complex scalar function $u_{p l}(r, z)$ describing the distribution of the field amplitude is given by

$$
\begin{aligned}
u_{p l}(r, z)= & (-1)^{p} \frac{C}{w(z)}\left(\frac{r \sqrt{2}}{w(z)}\right)^{l} L_{p}^{l}\left(2 r^{2} / w^{2}(z)\right) \times \exp \left[-i(2 p+l+1) \tan ^{-1}\left(z / z_{R}\right)\right] \\
& \times \exp \left[\frac{-i k r^{2} z}{2\left(z^{2}+z_{R}^{2}\right)}\right] \times \exp \left[-\left(r^{2} / w^{2}(z)\right)\right]
\end{aligned}
$$

Where $L_{p}^{l}$ are associated Laguerre polynomials, $z_{R}$ is the Rayleigh range, $w^{2}(z)=2\left(z_{R}{ }^{2}+z^{2}\right) / k z_{R}$ is the beam width at distance $z$ from the beam waist. The integer indices $l$ and $p$ are quantum numbers characterizing the mode and $l$ is the orbital angular momentum quantum number. From Eqs.(9) and (10), we obtain the expression of $\theta(\mathbf{R})$

$$
\theta(\mathbf{R})=-\frac{k r^{2} z}{2\left(z^{2}+z_{R}^{2}\right)}-l \phi-(2 p+l+1) \tan ^{-1}\left(z / z_{R}\right)-k z .
$$

In Eq.(7), the recoil shift (i.e. the second term) is so smaller than the Doppler shift in the first term that the recoil term can be not considered further. Therefore, we only consider the first term including the Doppler shift of the Laguerre-Gaussian beam from the three-level atom:

$$
\delta_{L G}=\left[-i \frac{\nabla f(\mathbf{R}, t) \cdot \mathbf{v}}{f(\mathbf{R}, t)}\right]_{0}=\delta_{L G}{ }^{(1)}-i \delta_{L G}{ }^{(2)} .
$$

Where 
$\delta_{L G}{ }^{(1)}=\left[\frac{f_{1}(\mathbf{R}, t) \nabla\left[\theta(\mathbf{R})+\Delta_{0} t\right] \cdot \mathbf{v}+f_{2}(\mathbf{R}, t) \nabla\left[\theta(\mathbf{R})+\left(\Delta_{0}+\Delta_{g}\right) t\right] \cdot \mathbf{v}}{f(\mathbf{R}, t)}\right]_{0}$

$\delta_{L G}^{(2)}=\left[\frac{\nabla G_{1}(\mathbf{R}) \cdot \mathbf{v} \times \exp i\left[\theta(\mathbf{R})+\Delta_{0} t\right]+\nabla G_{2}(\mathbf{R}) \cdot \mathbf{v} \times \exp i\left[\theta(\mathbf{R})+\left(\Delta_{0}+\Delta_{g}\right) t\right]}{f(\mathbf{R}, t)}\right]_{0}$

The imaginary part $\delta_{L G}{ }^{(2)}$ in Eq. (12) is determined by the change of the electric field amplitude of the LaguerreGaussian beam, which is not related with the final detuning or Doppler shift and is not considered further. The term $\delta_{L G}{ }^{(1)}$ can be further written as

$$
\delta_{L G}^{(1)}=\nabla \theta(\mathbf{R}) \cdot \mathbf{v}+\left(\Delta_{0}+\frac{D_{23}}{D_{13}+D_{23}} \Delta_{g}\right) \times(\nabla t \cdot \mathbf{v}) .
$$

In Eq.(14), the term $\Delta_{0}$ is the Laguerre-Gaussian beam detuning from the three-level atomic transition $\omega_{13}$. The term $\nabla \theta(\mathbf{R}) \cdot \mathbf{v}$ is the Doppler shift same as the result from the two-level atomic mode, which is given by [7]

$$
\begin{aligned}
\nabla \theta(\mathbf{R}) \cdot \mathbf{v}= & {\left.\left[-k+\frac{k r^{2}}{2\left(z^{2}+z_{R}^{2}\right)}\left(\frac{2 z^{2}}{\left(z^{2}+z_{R}^{2}\right.}\right)^{-1}\right)-\frac{(2 p+l+1) z_{R}}{\left(z^{2}+z_{R}^{2}\right)}\right] v_{z} } \\
& -\left(\frac{k z r}{z^{2}+z_{R}^{2}}\right) v_{r}-\left(\frac{l}{r}\right) v_{\phi}
\end{aligned}
$$

Where $v_{z}, v_{r}$ and $v_{\phi}$ are the axial, radial and azimuthal velocity components of the atom. We know from Eq.(14) that the detuning amending term $\Delta_{\mathrm{am}}=$ $D_{23} \Delta_{\mathrm{g}} /\left(\mathrm{D}_{13}+\mathrm{D}_{23}\right)$ results from the three-level mode, which is determined by two lower level split $\Delta_{\mathrm{g}}$ and the ratio of dipole matrix element $D_{23}$ to the total dipole matrix element $D_{23}+D_{13}$. From Eq.(14), we also find that there is same frequency amending for the axial, radial and azimuthal frequency detuning. Therefore, we can define an effective detuning of Laguerre-Gaussian beam from the three-level atom, which is given by

$$
\Delta_{e f f}=\Delta_{0}+\Delta_{a m}=\Delta_{0}+\frac{D_{23}}{D_{13}+D_{23}} \Delta_{g} .
$$

According to the effective detuning of LaguerreGaussian beam from the three-level atom, we also define an effective two level-atomic mode. In such effective two level atomic mode, the upper level is $|3\rangle$ and the lower level locates between levels $|1\rangle$ and $|2\rangle$ with a frequency interval $\Delta_{\mathrm{am}}$ from level $|1\rangle$. When $\Delta_{0}>>\Delta_{\mathrm{am}}$, that is the case of large detuning $\Delta_{0}$ compared with $\Delta \mathrm{g}$, we can neglect the amending frequency in the effective detuning. Therefore, the three-level atomic mode can be simplified as the two-level atomic mode. When the detuning $\Delta_{0}$ is about the order of $\Delta \mathrm{g}$, the detuning amending term $\Delta_{\mathrm{am}}$ plays an important role for the effective detuning and Doppler shift. In particular, when $\Delta_{0}=0$, the laser frequency is resonant with $\omega_{13}$, but the laser frequency is detuned $\Delta_{\mathrm{am}}$ from the effective two-level atomic mode. When $\Delta_{0}=-\Delta_{\text {am }}$, the laser frequency is resonant with the effective two-level atomic mode.

To discuss further quantitatively about the detuning amending term $\Delta_{\mathrm{am}}=\mathrm{D}_{23} \Delta_{\mathrm{g}} /\left(\mathrm{D}_{13}+\mathrm{D}_{23}\right)$, we consider the relation between $\Gamma$ and $\mathrm{D}[10]$

$$
\Gamma=\omega^{3} D^{2} /\left(3 \pi \varepsilon_{0} \hbar c^{3}\right),
$$

here $D$ is the dipole matrix element, $\Gamma$ is the spontaneous transition rate, $\omega$ is atomic resonance frequency, $\varepsilon_{0}$ is the free space permittivity, $\hbar$ is Planck constant over $2 \pi, c$ is the speed of light in vacuum, and we derive

$$
\Delta_{a m}=\frac{D_{23}}{D_{13}+D_{23}} \Delta_{g}=\frac{\omega_{13} \sqrt{\omega_{13} \Gamma_{23}}}{\omega_{13} \sqrt{\omega_{13} \Gamma_{23}}+\omega_{23} \sqrt{\omega_{23} \Gamma_{13}}} \Delta_{g} .
$$

Considering a hyperfine structure three-level ${ }^{85} \mathrm{Rb}$ atom, $|1\rangle \leftrightarrow 5 S_{1 / 2}, \quad F=2$ state, $|2\rangle \leftrightarrow 5 S_{1 / 2}, F=3$ state, $|3\rangle \leftrightarrow 5 P_{3 / 2}$ state, $\Delta_{\mathrm{g}}=3.04 \mathrm{GHz}, \lambda=0.78 \mu \mathrm{m}, \Gamma_{13}=0.74 \Gamma_{3}$, $\Gamma_{23}=0.26 \Gamma_{3}$, we calculate Eq.(18) and derive the approximate value of the amending term

$$
\Delta_{a m}=\frac{D_{23}}{D_{13}+D_{23}} \Delta_{g} \approx 0.37 \Delta_{g} \approx 1.12 \mathrm{GHz} .
$$

In particular, for $\lambda=0.78 \mu \mathrm{m}$ and $\nu=1 \mathrm{~m} / \mathrm{s}$, the axial Doppler shift $k v$ is about $1.3 \mathrm{MHz}$, which is much smaller than $\Delta_{a m}$, and radial and azimuthal Doppler shift are also about $\mathrm{MHz}$ much smaller than $\Delta_{\mathrm{am}}$ [7]. Therefore, the Doppler shift can be distinguished obviously from the detuning amending term. If the two lower levels are two magnetic sublevels and the level splitting $\Delta_{\mathrm{g}}$ can be modified in a wide range by changing magnetic field, we find the detuning amending term $\Delta_{\text {am }}$ can reach the order of Doppler shift. Therefore, the Doppler shift is easily affected by the detuning amending term $\Delta_{\mathrm{am}}$, which should be well distinguished from detecting the Doppler shift.

From Eqs. (7), (8.a), (14), we find that the effective detuning $\Delta_{\text {eff }}$ derived from the three-level atom in the plane beam or Laguerre-Gaussian beam is not related with the form of light field amplitude and phase, that is, the expression of the effective detuning in Eq.(16) is a general expression for every kind of the light field. The origination of the effective detuning is the three-level atomic mode.

\section{Conclusions}

In conclusion, we have derived the general analytic expression of the effective detuning $\Delta_{\text {eff }}=\Delta_{0}+D_{23} \Delta_{\mathrm{g}} /\left(D_{13}\right.$ $+D_{23}$ ) of the linearly polarized light field from the $\Lambda$ configuration three-level atomic mode. The detuning amending term $\Delta_{\mathrm{am}}=\mathrm{D}_{23} \Delta_{\mathrm{g}} /\left(\mathrm{D}_{13}+\mathrm{D}_{23}\right)$ resulting from the three-level mode is not related with the form of light field, which is decided by two lower level split $\Delta_{\mathrm{g}}$ and the ratio of dipole matrix element $\mathrm{D}_{23}$ to the total dipole matrix element $D_{23}+D_{13}$. For a linearly polarized Laguerre- 
Gaussian laser beam, we find that there is same frequency amending for the axial, radial and azimuthal frequency detuning. We define an effective two-level atomic mode in which the upper level is $|3\rangle$ and the lower level locates between levels $|1\rangle$ and $|2\rangle$ and has the frequency interval $\Delta_{\mathrm{am}}$ from level $|1\rangle$. The effective detuning plays an important role in detecting the laser detuning and Doppler shift from atomic transition frequency.

\section{ACKNOWLEDGEMENTS}

This research was supported by the National Key Basic Research Program of China (Grant No. 2011CB013004), the Natural Science Foundation of the Jiangsu Higher Institutions of China under Grant No. 11KJA460003, the Natural Science Foundation of the Jiangsu Province under Grant No. BK2011462, Jiangsu Province Key Laboratory funded project of the Photonic Manufacturing Science and Technology under Grant No.GZ201207.

\section{REFERENCES}

[1] Beth R A. Mechanical Detection and Measurement of the Angualar Momentum of Light. Physic Review, 50 ,pp.115, 1936.
[2] Allen L, Beijersbergen M W, Spreeuw R J C and Woerdman J P.Orbital angular momentum of light and the transformation of Laguerre-Gaussian laser mode. Physics Review. A, 45 pp.8185,1992.

[3] He H, Friese M E J, Heckenberg N R and Rubinsztein-Dunlop H.Direct observation of transfer of angular momentum to absorbing particles from a laser beam with a phase singularity. Physics Review Letters, 75, pp.826,1995.

[4] Friese M E J, Enger J, Rubinsztein-Dunlop H and Heckenberg N R. Optical angular momentum transfer to trapped absorbing particles. Physics Review A,54, pp.1593-1596,1996.

[5] Ambrosio A, Piccirillo B, Sasso A and Santamato E. Experimental and theoretical study of the transient rotation of isotropic transparent microparticles in astigmatic optical tweezers. Optics Communication, 230,pp.337-345,2004.

[6] Garces-Chavez V, Volke-Sepulveda K, Chavez-Cerda S, Sibbett W and Dholakia K. Transfer of orbital angular momentum to an optically trapped low-index particle. Physics Review A,66,063402,2002.

[7] Allen L, Babiker M and Power W L. Azimuthal doppler shift in light beams with orbital angular momentum . Optics Communication,112,pp.141,1994.

[9] Wang Zheng- Ling and Yin Jian-Ping.Analytic Solution of ThreeLevel Dressed-Atom Model. Chinese Physics Letters, 21, $1926,2004$.

[10] Metcalf H J and van der Straten P. Laser Cooling and Trapping. NewYork: Springer,1999. 